\title{
Prevención de infecciones nosocomiales: estrategias para mejorar la seguridad de los pacientes en la Unidad de Cuidados Intensivos
}

\author{
T. LISBOA ${ }^{a, b}$ Y J. RELLO \\ aServicio de Cuidados Intensivos. Hospital Universitario Joan XXIII. Universidad Rovira y Virgili.

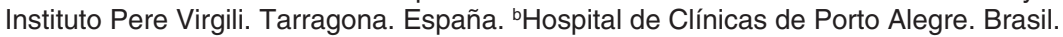

\begin{abstract}
La prevención de la infección nosocomial constituye una oportunidad de intervención para promover la seguridad de los pacientes. Las estrategias para obtener la mejora de las medidas de prevención disponibles son el principal desafió en la práctica clínica. El uso de paquetes de medidas, la formación continua, y las informaciones acerca del cumplimiento de las medidas propuestas son cruciales para mejorar la seguridad de los pacientes ingresados en las Unidades de Cuidados Intensivos.
\end{abstract}

PALABRAS CLAVE: $N A V$, seguridad, control infecciones, paquete de medidas.

\section{PREVENTION OF NOSOCOMIAL INFECTIONS: STRATEGIES TO IMPROVE THE SAFETY OF THE PATIENTS IN THE INTENSIVE CARE UNIT}

Infection prevention is an intervention opportunity to promote patient safety. The strategies to obtain the full implementation of available prevention measures is the main challenge in clinical practice. Using care bundles, continuing education and feedback on adherence to the measures

Financiado en parte por el Fondo de Investigaciones Sanitarias (CIBER 06/06/0036), AGAUR (2005/SGR/920) y FADO (Fundación Avedis-

Donabedian). FISS 07/90960

Correspondencia: Dr. J. Rello.

Servicio de Cuidados Intensivos.

Hospital Universitario Joan XXIII.

C/ Dr. Mallafre Guasch, 4.

43007 Tarragona. España.

Correo electrónico: jordi.rello@urv.ca

Correo electrónico: jrello.hj23.ics@ gencat.net

Manuscrito aceptado el 4-VI-2007. proposed are key points to improve the safety of patients in the Intensive Care Unit.

KEY WORDS: VAP, safety, infection control, care bundles.

\section{"Si buscas resultados distintos, no hagas siempre lo mismo". Albert Einstein (1879-1955)}

\section{INTRODUCCIÓN}

Las infecciones nosocomiales son la complicación mas común en pacientes hospitalizados. Aproximadamente una cuarta parte de estas infecciones ocurren en pacientes ingresados en Unidades de Cuidados Intensivos (UCI), aunque los cuidados intensivos no sean más que un $10 \%$ de la camas hospitalarias ${ }^{1}$. Esta complicación se adapta a la definición de acontecimiento adverso, que incluye acontecimientos no esperados o no deseados que ocurren asociados al cuidado al paciente $e^{2,3}$. Las infecciones son el acontecimiento adverso más frecuente, y afectan aproximadamente a 2 millones de personas en EE. UU., causando cerca de 100.000 muertes, con un coste asociado estimado en 5 billiones de dólares americanos al año ${ }^{4}$.

La definición de seguridad del paciente es variable en diferentes sitios y de acuerdo con diferentes autores. La definición más aceptable hoy en día es la que define la seguridad como la ausencia de potencial para la ocurrencia de lesiones asociadas al proceso de cuidado, generada por prevención de errores o de sus efectos ${ }^{3,5}$. Pero esto genera alguna confusión, y puede resultar complicado diferenciar los eventos relacionados a la seguridad con errores o 
complicaciones inevitables. Además, nuestra capacidad de medir y evaluar el efecto de intervenciones para mejorar la seguridad de los pacientes es extremamente limitada.

Las UCI han sido identificadas como un punto clave en el desarrollo de acontecimientos adversos. Trabajan con situaciones extremas, y su gran capacidad de salvar vidas está asociada con un gran riesgo de causar daños ${ }^{6}$. Por ello, constituyen un área de especial interés para el desarrollo de medidas de seguridad. Diversos programas han sido descritos con este objetivo, como implementación de equipos de respuesta rápida, hojas de flujos y checklists, aplicación de care bundles en grupos de enfermos como en la sepsis, y grupo de medidas para prevenir acontecimientos adversos relacionados con la infección como la neumonía asociada a la ventilación (NAV) y sepsis relacionada con catéter.

Shulman y $\mathrm{Ost}^{7}$ han discutido cómo están relacionados los conceptos referidos al control de infecciones y a la seguridad del paciente, y cómo las medidas que permiten el control/prevención de las infecciones, que son mesurables, tienen un impacto en cuestiones referidas a la seguridad.

La pregunta que surge es si las infecciones nosocomiales son acontecimientos adversos evitables, y, si lo son, de qué manera se puede intervenir en la cadena de eventos que culminan en su desarrollo para intentar minimizar su aparición y sus efectos. Esto se incorpora al concepto de seguridad y tiene efectos en la calidad del cuidado.

En este artículo revisaremos estudios que evaluarán las medidas y su implementación en relación con dos de los acontecimientos adversos infecciosos más importantes que se producen en cuidados intensivos: la NAV y la infección relacionada con el catéter.

\section{SEGURIDAD EN LA UCI}

Algunos estudios han demostrado que intervenciones realizadas con el objetivo de mejorar la seguridad del paciente crítico se asocian con una reducción de costes y morbimortalidad ${ }^{8-11}$. La evidencia ha demostrado que es importante la creación de una cultura de seguridad en el entorno de los cuidados intensivos, que permita un cambio en la percepción de los profesionales ${ }^{6,12}$. El gran desafío está en la implementación de las medidas en la práctica clínica. Diversos estudios ya han demostrado la distancia existente entre la disponibilidad de evidencia y la utilización de la evidencia en la práctica clínica ${ }^{13-15}$. Esto hace que algunos enfermos estén recibiendo un nivel de cuidados subóptimo o quizás insuficiente. Buscar maneras de aumentar la ejecución de las medidas probadamente eficaces en las diferentes situaciones es promover la seguridad de los enfermos. El desarrollo del concepto de care bundles o paquete de medidas se adapta muy bien a esta realidad. Su objetivo es trasladar las evidencias a la práctica, agrupando un paquete de medidas sencillas capaces de asegurar una aplicación uniforme de la mejor evidencia disponible. Las medi- das individuales se basan en la mejor evidencia disponible. Mientras la aplicación por separado de estas medidas mejora el cuidado del paciente, la aplicación conjunta del paquete de medidas (bundle) mejora la evolución ${ }^{16-18}$.

\section{SEGURIDAD Y CONTROL DE LAS INFECCIONES}

El cambio de cultura generado por las publicaciones, relacionando los errores y los acontecimientos adversos con la calidad y la seguridad, están cambiando también el control de las infecciones. La idea de que «muchas infecciones son inevitables y algunas pueden ser prevenidas» se ha cambiado por «todas las infecciones son potencialmente evitables mientras no se demuestre el contrario» ${ }^{19}$. El método de identificación de potenciales puntos clave en el proceso, que puedan generar medidas de prevención, utilizado en la práctica del estudio de la seguridad, puede y debe ser utilizado también en el control de las infecciones.

Jain et $\mathrm{al}^{20}$ han utilizado esta estrategia con el objetivo de reducir las tasas de infección nosocomial. Han empleado 4 intervenciones que fueron: a) discusiones multidisciplinarias; b) reevaluar la necesidad de cuidados intensivos diariamente; c) utilización de paquetes de medidas de prevención para NAV, infección del tracto urinario (ITU) e infección asociada a catéter, y d) cambio en la cultura de seguridad, aunque su efecto no ha podido ser medido. Con estas intervenciones sencillas se logró obtener una reducción de $58 \%$ en la incidencia de la NAV, el $48 \%$ en las bacteriemias asociadas a catéter y $37 \%$ en las infecciones de tracto urinario. Aunque no sea posible en el estudio identificar cuáles son las intervenciones más significativas, el cambio de actitud resultante de las intervenciones parece ser el punto clave en la obtención de estos resultados.

\section{INFECCIÓN RELACIONADA CON EL CATÉTER}

La infección relacionada a catéter es una enfermedad yatrógena con una incidencia de 80.000 episodios al año, una mortalidad de aproximadamente 20.000 muertes al año y un coste asociado que puede llegar hasta 2 billones de dólares americanos al año en EE. UU. ${ }^{21}$. En la literatura se han reconocido numerosas medidas de prevención efectivas en la reducción de las infecciones, pero la ejecución de estas medidas en la práctica parece ser el problema más relevante, existiendo gran diferencia entre la mejor evidencia disponible y la práctica clínica ${ }^{22,23}$. Las medidas más importantes incluyen el uso de barreras estériles durante la inserción, el uso de clorhexidina en el punto de inserción, utilización preferente de la vía subclavia frente a femoral y evitar el mantenimiento innecesario del catéter. Pero las medidas cruciales siguen siendo una higiene de manos adecuada, y los principios de control de infección en la prevención ${ }^{24}$. 


\section{TABLA 1. Intervenciones para prevenir la infección relacionada con el catéter}

\section{Intervenciones}

1. Programa educacional para aumentar el conocimiento acerca de las medidas basadas en la evidencia

2. Facilitación del material para la inserción del catéter

3. Cuestionar diariamente la necesidad de permanencia del catéter

4. Aplicación de un checklist durante la inserción del catéter

5. Que las enfermeras puedan suspender la inserción del catéter si se produce alguna violación de las guías

\section{TABLA 2. Paquete de medidas utilizado para la prevención de la infección relacionada con el catéter}

\author{
Medidas de prevención \\ 1. Higiene de manos \\ 2. Uso de barreras durante la inserción de catéter \\ 3. Uso de chlorohexidina en la asepsia \\ 4. Evitar la vía femoral \\ 5. Remoción de catéteres innecesarios
}

Adaptada de Pronovost $\mathrm{P}$, et $\mathrm{al}^{18}$.

Berenholtz et $\mathrm{al}^{25}$ han estudiado una serie de intervenciones para eliminar las infecciones asociadas a catéter en la UCI. Las medidas adoptadas por el equipo responsable para mejorar la calidad incluían cinco intervenciones (tabla 1). Las medidas sugeridas por las guías eran adoptadas, antes de la intervención, en sólo un $62 \%$ de los procedimientos. Con estas intervenciones de control de calidad, el estudio logró una reducción en la incidencia de la infección relacionada con el catéter de 11,3/1.000 días de catéter para 0/1.000 días de catéter durante el período de intervención, demostrando que las medidas basadas en evidencias, cuando son bien hechas, tienen un efecto en la prevención de acontecimientos adversos. Además, los autores estiman que han evitado 43 episodios de infección relacionada con el catéter, 8 muertes y un coste adicional de cerca de 2 millones de dólares.

Asimismo, Warren et $\mathrm{al}^{26}$ han obtenido una reducción de $40 \%$ en la incidencia de las infecciones relacionadas con el catéter, con una intervención de educación, cuyo objetivo era poner de relieve las medidas basadas en la evidencia asociadas a la prevención de las infecciones asociadas al catéter. Warren et $\mathrm{al}^{27}$ han adoptado medidas educacionales, así como la incorporación en la práctica de las medidas basadas en la evidencia sugeridas por las guías con carácter multicéntrico, obteniendo resultados parecidos, con una reducción del $21 \%$ en la incidencia de las infecciones asociadas a catéter, pero con variabilidad entre los diferentes centros. El impacto de las medidas de educación en la reducción de la incidencia de infecciones es sostenida por otros trabajos ${ }^{23,28,29}$, y su importancia en la promoción de la seguridad de los pacientes sugiere la necesidad de su puesta en práctica.

Recientemente, Pronovost et $\mathrm{al}^{18}$ han evaluado un paquete de medidas (tabla 2) destinado a mejorar el cumplimiento de las recomendaciones del Center for Disease Control (CDC) acerca de la prevención de la infección relacionada con catéter. La puesta en mar- cha de las medidas se ha hecho a través de la educación, uso de checklist, discusión diaria de las necesidades y de la permanencia de los catéteres, interrupción del procedimiento si no se cumplen las recomendaciones y comunicación de los resultados a los participantes del estudio. Además de obtener una reducción, en los primeros 3 meses, de 2,7 a 0 episodios por cada 1.000 catéteres al día, el efecto se mantuvo durante los 18 meses de seguimiento, con una reducción de 7,7 a 1,4 episodios por cada 1.000 catéteres al día. La reducción en la incidencia de infecciones relacionadas con catéter, además de reducir los costes, tiene un efecto directo en la seguridad de los pacientes. Intervenciones que disminuyen el riesgo de acontecimientos adversos y demuestran un compromiso diario con la seguridad del paciente pueden ser un medio de mejorar los resultados ${ }^{30}$.

\section{NEUMONÍA ASOCIADA A LA VENTILACIÓN}

La NAV es la complicación infecciosa más importante en la UCI, y se corresponde con cerca de un $25 \%$ de las infecciones producidas en la $\mathrm{UCI}^{31,32}$. El coste anual en EE. UU. puede llegar a los 10 billones de dólares ${ }^{33}$. Aunque hay numerosas medidas de prevención de la NAV, bien descritas y evaluadas, la mayor parte siguen sin aplicarse. La disponibilidad de evidencias de medidas que pueden reducir la incidencia de la NAV no se traduce en cambios en la práctica diaria. Collard et al han publicado una revisión sistemática en $2003^{34}$, en la que identifican que la posición semi-incorporada, el uso de sucralfato en enfermos de bajo o moderado riesgo para profilaxis de sangrado gastrointestinal, la aspiración de secreciones subglóticas, y el uso de camas oscilantes son medidas eficaces en la prevención de la NAV. La ejecución de estas medidas no es uniforme. Se han evaluado las causas de la no adherencia de médicos y enfermeras $^{35-37}$, sugiriendo la necesidad de programas de educación que estimulen la consumación de las medidas.

Salahuddin et $\mathrm{al}^{38}$ han utilizado un programa de educación con el objetivo de reducir la incidencia de NAV. El programa de educación ha seleccionado medidas recomendadas en las guías, logrando una reducción de un $51 \%$ en la incidencia de la NAV. Resar et al ${ }^{17}$ ha utilizado un paquete de medidas (tabla 3), y ha encontrado una reducción del $45 \%$ en la incidencia de la NAV con la adopción de este paquete. Estas medidas fueron propuestas en la campaña del Institute for 
TABLA 3. Paquete de medidas para la prevención de la neumonía asociada a la ventilación (NAV)

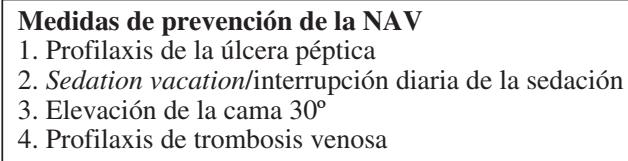

Adaptada de Resar R, et al ${ }^{17}$

Health Improvement (IHI) para salvar 100.000 vidas (www.ihi.org). Esta campaña tenía el objetivo de salvar 100.000 vidas con la ejecución de medidas de seguridad, y entre las medidas se encontraba la prevención de la NAV. Los centros participantes en la campaña han obtenido una importante reducción de la incidencia de la NAV $\mathrm{V}^{39.41}$.

El desarrollo del paquete de medidas permite una mejor puesta en marcha de las medidas de prevención probadamente eficaces, y una práctica más uniforme de acuerdo con la mejor evidencia disponible. Además, el uso de paquetes de medidas, no sólo en la prevención sino también en el diagnóstico y tratamiento de la NAV, constituye un amplio campo de investigación inexplorado. Es probable que la oportunidad para mejorar los resultados no venga del descubrimiento de nuevos tratamientos, sino de ejecución más efectiva de los existentes ${ }^{42}$.

En Cataluña, una propuesta del Departamento de Salud y de la Fundación Avedis-Donabedian está evaluando en 12 hospitales los efectos de aplicar un paquete de medidas simple (tabla 4) para incrementar la seguridad reduciendo la incidencia de la NAV. Los resultados de Pronovost ${ }^{17,18}$ en EE. UU. deben servir de ejemplo en otras áreas geográficas. En 2007 ha concluido un proyecto europeo multidisciplinar, con participación de expertos de 11 países ${ }^{43}$, para desarrollar un enfoque pan-europeo en la prevención y el manejo de la NAV. Ha llegado el momento de desarrollar un proyecto cooperativo que incremente la seguridad, centrado en la prevención de infecciones en las UCI de Latinoamérica.

\section{CONCLUSIÓN}

La diseminación de una cultura de seguridad centrada en la prevención en la línea del IHI (con la campaña de las 100.000 vidas y ahora con la campaña para evitar 5.000.000 de daños) prevendrá lesiones y muertes evitables en la UCI. Esto implica un cambio de cultura, pasando de recomendar largas listas de medidas a una relación de 3-5 medidas sencillas que deberán aplicarse simultáneamente.

Estos programas deben basarse en evidencias aplicadas y aceptadas en forma multidisciplinar. La formación continua y la información sobre el grado de cumplimiento son cruciales para mantener su eficacia. Si queremos mejor seguridad y menos daños colaterales, creemos que ha llegado el momento de poner en marcha paquetes de medidas, modificando las prácticas habituales.
TABLA 4. Paquete de medidas para la prevención de la neumonía asociada a la ventilación (NAV) en un estudio en Cataluña

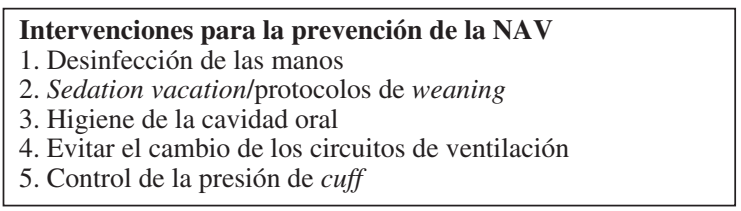

\section{Declaración de conflicto de intereses}

Los autores han declarado no tener ningún conflicto de intereses.

\section{BIBLIOGRAFÍA}

1. Eggimann P, Pittet D. Infection control in the ICU. Chest. 2001;120(6):2059-93.

2. Martin MC, Ruiz J. Adverse events in intensive medicine. Managing risk. Med Intensiva. 2006;30(6):284-92.

3. Pronovost PJ, Thompson DA, Holzmueller CG, Lubomsky LH, Morlock LL. Defining and measuring patient safety. Crit Care Clin. 2005;21:1-19.

4. Burke JP. Infection control $-\mathrm{A}$ problem for patient safety. $\mathrm{N}$ Engl J Med. 2003;348:651-6.

5. Vincent $C$. Understanding and responding to adverse events. N Engl J Med. 2003;348:1051-6.

6. Winters B, Dorman T. Patient-safety and quality initiatives in the intensive care unit. Curr Opin Anesthesiol. 2006;19:140-5.

7. Shulman L, Ost D. Managing infection in the critical care unit: how can infection control make the ICU safe? Crit Care Clin. 2005;21:111-28.

8. Zhan C, Miller MR. Exceso length of stay, charges and mortality attributable to medical injuries during hospitalization. JAMA. 2004;290:1868-74.

9. Hayward R, Hofer TP. Estimanting hospital deaths due to medical errors; preventability is in the eye of the reviewer. JAMA. 2004;286:415-20.

10. Berenholtz S, Pronovost P, Lipsett P, Dawson P, Dorman T. Assessing the effectiveness of critical pathways on reducing resource utilization in the surgical intensive care unit. Intensive Care Med. 2001;27:1029-36.

11. Pronovost PJ, Rinke ML, Emery K, Dennison C, Blackledge $\mathrm{C}$, Berenholtz SM. Interventions to reduce mortality among patients treated in intensive care units. J Crit Care. 2004;19:158-64.

12. Brennan TA, Gawande A, Thomas E, Studdert D. Accidental deaths, saved lives and improved quality. N Engl J Med. 2005; 353:1405-9.

13. Cabana MD, Rand CS, Powe NR, Wu AW, Wilson MH, Abboud PA, et al. Why don't physicians follow clinical practice guidelines? JAMA. 1999;282:1458-65.

14. Levy MM, Pronovost PJ, Dellinger RP. Sepsis change bundles: converting guidelines into meaningful change in behaviour and clinical outcome. Criti Care Med. 2004;32:595-7.

15. Yealy DM, Auble TE, Stone RA. Effect of increasing the intensity of implementing pneumonia guidelines: a randomized, controlled trial. Ann Intern Med. 2005;143:881-94.

16. Rivers E, Nguyen B, Havstad S, Ressler J, Muzzin A, Knoblich B, et al. Early goal-directed therapy in the treatment of severe sepsis and septic shock. N Engl J Med. 2001;345:1368-77.

17. Resar R, Pronovost P, Haraden C, Simmnonds T, Rainey T, Nolan T. Using a bundle approach to improve ventilator care processes and reduce ventilator-associated pneumonia. Jt Comm J Qual Patient Saf. 2005;31:243-8.

18. Pronovost P, Needham D, Berenholtz S, Sinopoli D, Chu H, Cosgrove $S$, et al. An intervention to decrease catheter-related bloodstream infections in the ICU. N Engl J Med. 2006;355:2725-32. 
19. Gerberding JL. Hospital-onset infections: a patient-safety issue. Ann Intern Med. 2002;137:665-70.

20. Jain M, Miller L, Belt D, King D, Berwick DM. Decline in ICU adverse events, nosocomial infections and cost through a quality improvement initiative focusing on teamwork and culture change. Qual Saf Health Care. 2006;15:235-9.

21. Mermel LA. Prevention of intravascular catheter-related infections. Ann Intern Med. 2000;132:391-402.

22. Saint S, Lipsky BA. Preventing catheter-related bacteriuria: should we? Can we? How? Arch Intern Med. 1999;159:800-8.

23. Sherertz RJ, Ely EW, Westbrook DM, Gledhill KS, Streed SA, Kiger B, et al. Education of physicians-in-training can decrease the risk for vascular catheter infection. Ann Intern Med. 2000; 132:641-8.

24. Saint S, Savel RH, Matthay MA. Enhancing the safety of critically ill patients by reducing urinary and central venous catheterrelated infections. Am J Respir Crit Care Med. 2002;165:1475-9.

25. Berenholtz SM, Pronovost PJ, Lipsett PA, Hobson D, Earsing K, Farley JE, et al. Eliminating catheter-related bloodstream infections in the intensive care unit. Crit Care Med. 2004;32: 2014-20.

26. Warren DK, Zack JE, Mayfield JL, Chen A, Prentice D, Fraser VJ, et al. The effect of an education program on the incidence of central venous catheter-associated bloodstream infection in a medical ICU. Chest. 2004;126:1612-8.

27. Warren DK, Cosgrove SE, Diekema DJ, Zuccoti G, Climo MW, Bolon MK, et al. A multicenter intervention to prevent catheter-associated bloodstream infections. Infect Control Hosp Epidemiol. 2006;27:662-9.

28. Eggimann P, Harbarth S, Constantin MN, Touveneau S, Chevrolet JC, Pittet D. Impact of a prevention strategy targeted at vascular-access care on incidence of infections acquired in intensive care. Lancet. 2000;355:1864-8

29. Cohran J, Larson E, Roach H, Blane C, Pierce P. Effect of intravascular surveillance and education program on rates of nosocomial bloodstream infections. Heart Lung. 1996;25:161-4.

30. Wenzel RP, Edmond MB. Team-based prevention of catéter-related infections. N Engl J Med. 2006;355:2781-3.

31. Kollef MH, Shorr A, Tabak YP, Gupta V, Liu LZ, Johannes RS. Epidemiology and outcomes of health-care-associated pneumonia: Results from a large US database of culture-positive pneumonia. Chest. 2005;3854-62.
32. The American Thoracic Society and the Infectious Diseases Society of America Guideline Committee. Guidelines for the management of adults with hospital-acquired, ventilator-associated, and healthcare-associated pneumonia. Am J Respir Crit Care Med. 2005; $171: 388-416$

33. Rello J, Ollendorf DA, Oster G, Vera-Llonch M, Bellm L, Redman R, et al. Epidemiology and outcomes of ventilador-asociated pneumonia in a large US database. Chest. 2002;122:2115-21.

34. Collard HR, Saint S, Matthay MA. Prevention of ventilatorassociated pneumonia: an evidence-based systematic review. Ann Intern Med. 2003;138:494-501.

35. Rello J, Lorente C, Bodi M, Diaz E, Ricart M, Kollef MH Why do physicians not follow evidence-based guidelines for preventing ventilator-associated pneumonia? A survey based on the opinions of an international panel of intensivists. Chest. 2002;122 656-61.

36. Ricart M, Lorente C, Díaz E, Kollef MH, Rello J. Nursing adherence with evidence-based guidelines for preventing ventilator-associated pneumonia. Crit Care Med. 2003;31:2693-6.

37. Rello J, Koulenti D, Blot S, Sierra R, Diaz E, De Walle JJ, et al. Oral care practices in intensive care units: a survey of 59 European ICUs. Intensive Care Med. 2007; doi 10.1007/s00134007-0605-3.

38. Salahuddin N, Zafar A, Sukhyani L, Rahim S, Noor MF, Hussain K, et al. Reducing ventilator-associated pneumonia rates through a staff education programme. J Hosp Infect. 2004;57:223-7.

39. Craven D. Preventing ventilador-associated pneumonia in adults: Sowing seeds of change. Chest. 2006;130:251-60.

40. Babcock HM, Zack JE, Garrison T, Trovillion E, Jones M, Fraser VJ, et al. An educational intervention to reduce ventiladorassociated pneumonia in an integrated health system: a comparison of effects. Chest. 2004; 125:2224-31.

41. Cocanour CS, Peninger M, Domonoske BD, Li T, Wright B, Valdivia A, et al. Decreasing ventilador associated pneumonia in a trauma ICU. J Trauma. 2006;61:122-9.

42. Pronovost PJ, Nolan T, Zeger S, Miller M, Rubin H. How can clinicians measure safety and quality in acute care? Lancet. 2004;363:1061-7.

43. Masterton R, Craven D, Rello J, Struelens M, FrimodtMoller N, Chastre J, et al. Hospital acquired pneumonia guidelines in Europe: a review of their status and future development. JAC. En prensa 2008. 